ENCYCLOPÉDIE Encyclopédie berbère

BERBERE

3 | 1986

3 | Ahaggar - Alī ben Ghaniya

\title{
Akarit (oued)
}

\section{Gragueb}

\section{OpenEdition}

Journals

Édition électronique

URL : http://journals.openedition.org/encyclopedieberbere/2394

DOI : 10.4000/encyclopedieberbere.2394

ISSN : 2262-7197

\section{Éditeur}

Peeters Publishers

\section{Édition imprimée}

Date de publication : 1 juillet 1986

Pagination : 409-413

ISBN : 2-85744-260-2

ISSN : 1015-7344

\section{Référence électronique}

Gragueb, « Akarit (oued) », Encyclopédie berbère [En ligne], 3 | 1986, document A139, mis en ligne le 01 décembre 2012, consulté le 13 octobre 2020. URL : http://journals.openedition.org/ encyclopedieberbere/2394; DOI : https://doi.org/10.4000/encyclopedieberbere.2394

Ce document a été généré automatiquement le 13 octobre 2020

(c) Tous droits réservés 


\section{Akarit (oued)}

\section{Gragueb}

1 Cet important gisement, situé à une trentaine de kilomètres au nord de la ville de Gabès, ne cesse depuis plus d'un demi-siècle d'attirer de nombreux spécialistes des sciences de la terre et de la Préhistoire.

2 Il occupe, en effet, une position centrale dans le golfe de Gabès et présente des séquences stratigraphiques, associées à des industries préhistoriques, représentatives de différentes phases du Quaternaire continental. C'est donc avec raison qu'on doit considérer ce gisement comme un des hauts lieux de la Préhistoire tunisienne.

\section{Historique des recherches}

3 «Marcel Solignac, chef du service géologique de l'administration tunisienne, nous a signalé une nouvelle station de surface qui porte les limites d'extension orientale de l'Oranien fort au delà du point extrême jusqu'ici connu ». C'est en ces termes que le Dr. E. G. Gobert s'exprimait pour annoncer au monde savant la découverte d'industries préhistoriques à Oued Akarit. Il s'agissait du gisement moustérien coupé par le chenal actuel de l'oued et de la station A qui surmonte une dune environnante.

4 La fouille du gisement moustérien a été menée en octobre-novembre 1951 (Gobert E. G. 1952, p. 225). Au cours de cette fouille R. Schmalz a repéré la station C (Gobert E. G. 1952, p. 228). Au début de l'année suivante E. G. Gobert, accompagné des membres de l' American school of prehistoric research, étudie le site et effectue des ramassages sur la station A et des fouilles dans les stations B et C (Gobert et Howe, 1955). Entre 1954 et 1955 (Gobert, Libyca, 1955, p. 394) le Dr. Gobert reprend, avec G. Laplace, la fouille de « trois niveaux à lamelles ».

Dans les années qui suivent, R. Coque, en compagnie du Dr. Gobert, visite le site et le place dans le schéma général du Quaternaire continental du Sud tunisien. En 1968, une équipe américaine de l'Université du Colorado y effectue des recherches, surtout stratigraphiques (Page, 1972). 
6 L'Oued Akarit continue toujours à attirer un nombre important de spécialistes des sciences de la terre. Ainsi, tout récemment, une étude consacrée à la stratigraphie quaternaire du golfe de Gabès donne à 0 . Akarit un rôle de premier plan (Ben Ouezdou, 1984). Les datations radiométriques ne manquent pas d'apporter leur secours et les toutes dernières ont été publiées en 1983 (Fontes et al. 1983).

\section{Cadre morphologique et stratigraphique}

Bien que situé à $4 \mathrm{~km}$ de la mer, le site de Oued Akarit ne recèle aucune trace du Quaternaire marin.

8 A l'arrivée des Moustériens le site de Oued Akarit offrait le paysage d'une lagune alimentée par les sources du Continental intercalaire dont l'écoulement vers la mer était bloqué par un cordon littoral en rapport avec les derniers soubresauts du mouvement transgressif tyrrhénien. L'édification du glacis 2 commence à ce moment.

$\mathrm{Au}$ cours de la phase suivante des cônes alluviaux s'accumulent, sur lesquels viennent s'installer des hommes de la station C. La mer était éloignée de la ligne de côte actuelle. Une phase d'aridité s'installe par la suite au cours de laquelle une croûte gypseuse recouvre les industries à lamelles de la station $\mathrm{C}$.

Oued Akarit. Croquis de situation des différents gisements

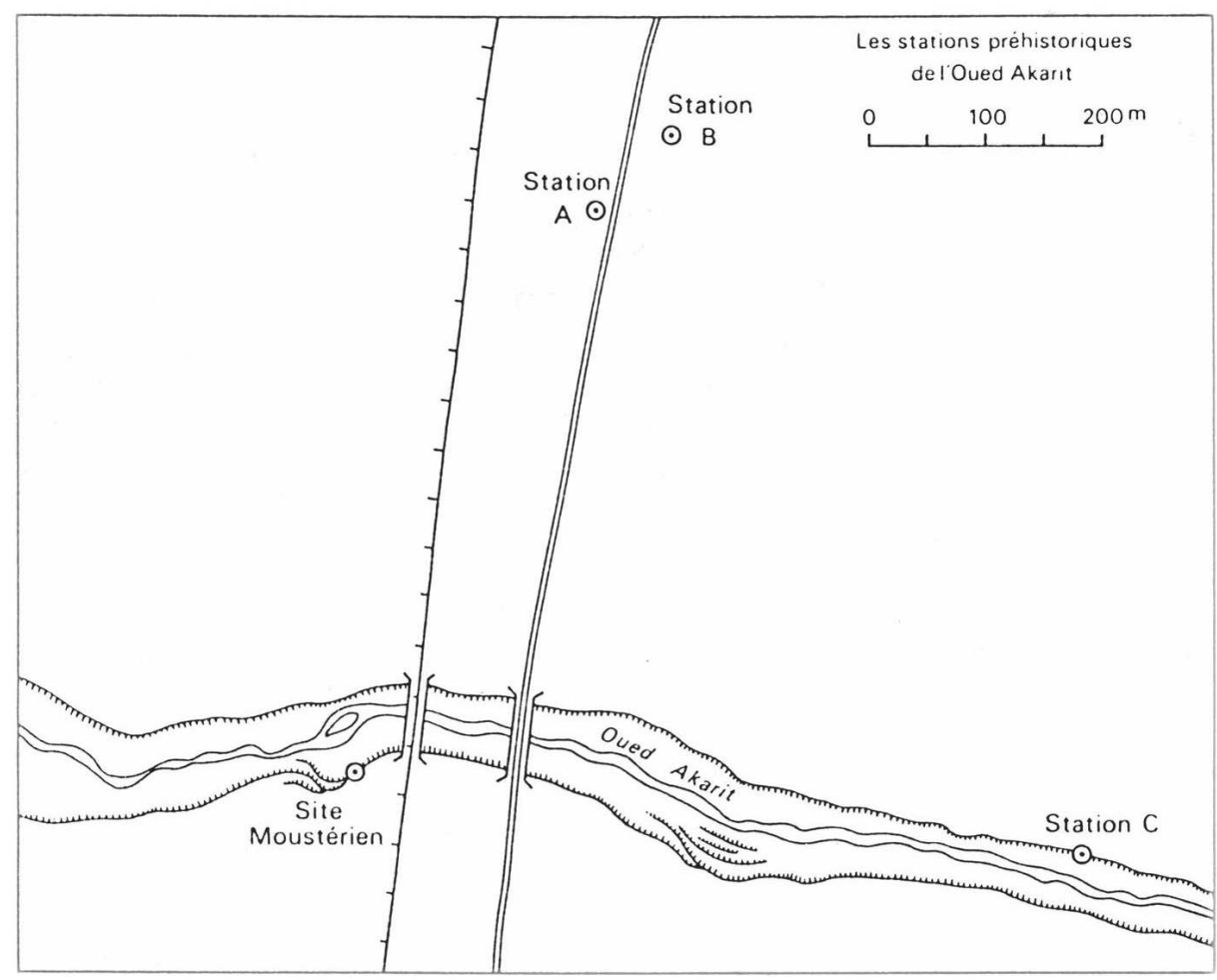

10 A la phase suivante l'oued Akarit creuse son chenal et des dunes s'installent de part et d'autre de ce chenal.

11 Les hommes de la station B s'établissent postérieurement à la constitution de la croûte gypseuse et sont contemporains de l'accumulation dunaire (leur industrie se trouve à la 
base de la dune). Les lamelles de la station A couvrent la dune et sont donc contemporaines d'un paysage qui n'a point changé depuis.

Les études stratigraphiques menées à Oued Akarit (Page 1972 et Ben Ouezdou 1983) montrent la succession suivante :

1. Argiles et limons gypseux coupés par des niveaux hydromorphes tourbeux auxquels est associée une industrie moustérienne. Cette couche est appelée "Akarit Formation » par W. D. Page et « membre Akarit I » par H. Ben Ouezdou.

2. Niveau d'érosion qui contient des galets de croûte gypseuse (niveau «Alpha » de W. Page).

3. Dépôt limoneux rougeâtre dont l'épaisseur varie entre 4 et $5 \mathrm{~m}$. On y trouve, localement, un lit de Cardium en position de vie passant latéralement à un dépôt grisâtre qui constitue la couche archéologique contenant les industries à lamelles de la station C.

4. Croûte gypseuse blanchâtre contenant un pourcentage inhabituel de sables quartzeux très fins. Dépôt limoneux et croûte gypseuse constituent la «Demna formation» de W. D. Page ou le «membre Akarit II» de H. Ben Ouezdou. Le même facies alluvial a été observé sur les berges de l'oued Demna (18 $\mathrm{km}$ au sud) qui lui a donné son nom.

5. Limons et sables fins d'origine dunaire auxquels sont associées les industries de la station A et de la station B.

\section{Chronologie absolue}

Les dépôts quaternaires de Oued Akarit ont bénéficié de nombreuses datations radiométriques. W.D. Page (1972) obtient 6 datations, deux dans la " Akarit formation» (niveau moustérien): $26150 \pm 1300$ B.C. et 20550 B.C. et quatre dans la « Demna formation » (les deux premières sont dans la lagune à Cardium) : $6285 \pm 180$ B.C. ; 6485 \pm 180 B.C. ; $6685 \pm 260$ B.C. ; $7235 \pm 210$ B.C.

14 Paskoff R. et Sanlaville P. (Ben Ouezdou 1983, p. 170) datent des cardium de lagune et obtiennent $6290 \pm 170$ B.C. Deux géomorphologues allemands obtiennent en 1975 (Brosche et Molle 1975) des datations radiométriques pour des niveaux dont le facies de sédimentation correspond à celui de l'o. Akarit. Ces niveaux ont été identifiés dans des berges d'oueds situées au sud de Gabès, O. Zigzaou, O. el Hallouf, etc.

Ils distinguent une "main accumulation » qui doit correspondre au niveau «Akarit formation » et une « younger accumulation » qui doit correspondre au niveau « Demma formation ». Pour le niveau ancien ils donnent: $19435 \pm 235$ B.C. et pour le niveau récent ils donnent : $6650 \pm 150$ B.C. $-5825 \pm 340$ B.C.

Steinmann S. et Bartels G. (1982) datent un niveau dans l'oued M'dou (sortie sud de Gabès) qui correspond au sommet du glacis 2. Ils obtiennent : $5320 \pm 155$ B.C.

Une étude de paléohydrologie menée par Fontes, Coque et al. (1983) a permis d'effectuer près de 29 datations sur des matières organiques et des carbonates de l'o. Akarit. De ces datations nous retenons celle de la croûte gypseu-se qui surmonte le glacis $2: 4045$ B.C. Pour les formations plus anciennes, Formation Akarit et Formation Demna, les datations de Fontes confirment dans l'ensemble celles obtenues par W. Page et ses successeurs. 
18 Ainsi, de multiples datations, dans différents endroits, et effectuées par différents laboratoires, donnent une grande fiabilité à la chronologie avancée par W. D. Page. Nous pouvons résumer cette chronologie ainsi : entre 25000 et $20000 \mathrm{av}$. J.-C, des hommes moustériens fréquentaient les sources du site de Oued Akarit. Entre 7000 et 6 000 les hommes de la station $\mathrm{C}$ viennent fréquenter les rives de la lagune à Cardium. Vers 4000 av. J.-C. une croûte gypseuse est venue sceller lamelles et cardium. Au cours du IIIe millénaire les hommes de la station B et A s'installent : les uns sur la croûte, les autres sur la dune.

19 La date avancée pour le Moustérien semble trop récente pour être acceptée par les préhistoriens. Nous aurions alors à 0 . el Akarit un moustérien d'âge Atérien final...! Mais, que connaissons-nous de la chronologie du Moustérien en Tunisie et en Afrique du Nord ? Il faut avouer que c'est peu de choses. Il serait donc prématuré de rejeter en bloc cette chronologie car nous ne devons pas ignorer les problèmes que pose le Moustérien en Afrique du Nord (Balout L. 1955 et 1965); il est hors de notre propos d'entrer dans la polémique qui anime ce brûlant sujet.

Que faut-il penser de la date avancée pour les lamelles? Nous l'acceptons dans la mesure où cette date concorde avec celle de certains faciès du Sud algérien (El Haouita, El Hamel, El Hadjar, etc.). Elle est d'autant plus acceptable que les industries de l'oued Akarit présentent de troublantes ressemblances avec les industries d'Aïn Naga (Capsien supérieur). Or les datations obtenues pour Aïn Naga se situent entre 7350 B.C. et 6950 B.C. (Grébénart 1969).

\section{Typologie}

21 Par leur facies typologique et leur chronologie les industries de O. Akarit seraient des industries que l'on pourrait classer dans un Capsien supérieur de faciès méridional (Camps G. 1974). De toutes les industries à lamelles du Sud tunisien, O. Akarit se distingue par la relative faiblesse de la valeur indiciaire de ses lamelles et l'indice anormalement élevé de ses microlithes géométriques et de ses microburins. C'est un monde à part...

Le moustérien, lui, ne manque pas d'originalité. Déjà, en 1933, le Dr. Go-bert envisage de le qualifier d'Atérien « lorsqu'on le connaîtra mieux » (Gobert 1933, p. 649). En 1962 il nuance sa définition et le qualifie de "moustérien léger, sans pierres épaisses " (Gobert 1962, p. 274).

\section{Faune}

Une faune de vertébrés plus ou moins abondante a été découverte dans les niveaux moustériens. Il s'agit de: Equus mauritanicus, Rhinoceros simus, Camelus Thomasi Pomel (Gobert 1962). Par ailleurs, de nombreux gîtes riches en malacofaunes et en microfaunes ont été découverts en aval et en amont des ponts. Des études récentes (Lévy 1983) montrent qu'il s'agit d'espèces et d'associations semblables à celles qui peuplent les milieux margino-littoraux du golfe du Lion et qui sont nées de l'interaction d'influences thalassoïdes et continentales. Ces résultats conduisent à une nouvelle interprétation de l'évolution paléogéographique du Sud tunisien; interprétation qui envisage un jeu tectonique récent qui aurait provoqué une surrection appréciable du 
continent. Or rien, dans la morphologie ou la tectonique, ne vient appuyer cette hypothèse (Coque 1962.) Ainsi, la géologie et la paléontologie avancent des hypothèses contradictoires et font revivre la vieille polémique sur une éventuelle communication entre les dépressions fermées du Sud tunisien et le golfe de Gabès. Par ses gisements préhistoriques, par ses gisements fossilifères et par les problèmes scientifiques qu'il pose, Oued Akarit est un site de choix aussi bien pour la préhistoire que pour la paléogéographie.

\section{BIBLIOGRAPHIE}

BALOUT L. Préhistoire de l'Afrique du Nord. Essai de chronologie. Paris, A.M.G. 1955.

BALOUT L. Le Moustérien du Maghreb, Quaternaria, Rome, 1965, 7, p. 43-58.

BELLAICHE G. et BLANPIED G, Evolution sédimentaire quaternaire de la plateforme pélagienne. Géologie méditerranéenne, La mer pélagienne, Aix en Provence, 1979, p. 304-307.

BEN OUEZDOU H. Etude géomorphologique et stratigraphique des formations quaternaires dans les alentours du golfe de Gabès. Thèse de 3e cycle, Tunis, 1983, $213 \mathrm{p}$.

BEN OUEZDOU H. Stratigraphie des dépôts quaternaires continentaux du golfe de Gabès (Sud tunisien), C.R. Acad. Sc. Paris, 1984, t. 299, sér. II, n 19, p. 1351-1354.

BEN OUEZDOU H., CAMPS G., GRAGUEB A., MAHJOUB K., et zOUARI K. Sur les dépôts du Pléistocène supérieur et de l'Holocène de la région des Chotts et de la plaine côtière du Golfe de Gabès (Tunisie) et leur place dans la stratigraphie du quaternaire. CR. Acad. Sc. Paris, t. 302, série II, n. ${ }^{\circ}$ 9, 1986, p. 659-669.

BROSCHE V. K. et MOLLE H. G. Morphologische Untersushungen im nordöstliche Matmata-Volland... Eszeitalter V, 1975, Gegenwart, Seite 218-240.

CAMPS G. Les civilisations préhistoriques de l'Afrique du Nord et du Sahara. Paris, 1974, Doin. COQUE R. La Tunisie présaharienne, Etude géomorphologique. Paris, 1962.

FONTES J. C, COQUE R., DEVER L., FILlY A. ET MAMOU A. Paléohydrologie isotopique de l'oued el Akarit (Sud Tunisie) au Pléistocène et à l'Holocène, Palaeogeography, palaeoclimatology, palaeoecology, Amsterdam, 1983, n 43, p. 41-62.

GOBERT E. G. L'Oranien dans la région de Gabès, l'Anthropologie, 1933, t. XLIII, p. 649.

GOBERT E. G. Notions générales acquises sur la préhistoire de la Tunisie. Actes du IIe Congr. panafr. de Préhist., Alger 1952 (1955), p. 221-239.

GOBERT E. G. et HOWE B., L'Ibéromaurusien de l'oued el-Akarit (Tunisie), Actes du IIe congrès panafr. Alger 1952 (1955), p. 575-594.

GOBERT E. G. et HOWE B. La Préhistoire dans la zone littorale de la Tunisie, Quaternaria (Rome), 6, 1962, p. 271-307. 
GREBENART D. Aïn Naga, Capsien et Néolithique des environs de Messad, Libyca, t. 17, 1969, p. 93-197.

LEVY A. Données nouvelles sur la paléogéographie du sud tunisien au quaternaire supérieur, Benthos, 1983, p. 370-379.

LEVY A. Les associations margino-littorales de foraminifères et d'organismes associés de quelques gisements quaternaires du site de l'oued el Akarit (sud tunisien), Benthos, 1983, p. 361-367.

PAGE W. D. The geological setting of the archaeological site at O. el Akarit and the palaeoclimatic significance of gypsum soils, Southern tunisia, Thesis, Univ. of Colorado, 1972, 111 p.

ROGNON P., LEVY A., BALLAIS J.-L., GOUDE G, et RISER J. Enai d'interprétation des coupes du quaternaire récent de l'oued el Akarit (Sud Tunisie), Geologie méditerranéenne, t. 10, 1983, p. 71-91.

STEINMANN S. et BARTELS G. K. Quatärgeomorphologische beobachtungen aus nord und süd tunesien, Catena $1,9,1982$, p. 95-108.

INDEX

Mots-clés : Préhistoire, Tunisie 